\title{
Improving the prediction of the Madden-Julian Oscillation of the ECMWF model by post-processing
}

\author{
Riccardo Silini ${ }^{1}$, Sebastian Lerch ${ }^{2}$, Nikolaos Mastrantonas ${ }^{3,4}$, Holger Kantz ${ }^{5}$, Marcelo Barreiro ${ }^{6}$, and \\ Cristina Masoller ${ }^{1}$ \\ ${ }^{1}$ Departament de Física, Universitat Politècnica de Catalunya, Sant Nebridi 22, 08222 Terrassa, Barcelona, Spain. \\ ${ }^{2}$ Institute of Economics, Karlsruhe Institute of Technology, Blücherstr. 17, 76185 Karlsruhe, Germany. \\ ${ }^{3}$ European Centre for Medium-Range Weather Forecast (ECMWF), Reading, UK. \\ ${ }^{4}$ Technische Universität Bergakademie Freiberg (TUBAF), Freiberg, Germany. \\ ${ }^{5}$ Max-Planck Institute for the Physics of Complex Systems, 01187 Dresden, Germany. \\ ${ }^{6}$ Departamento de Ciencias de la Atmósfera, Facultad de Ciencias, Universidad de la República, Igua 4225, 11400 \\ Montevideo, Uruguay.
}

Correspondence: Riccardo Silini (riccardo.silini@ outlook.com)

\begin{abstract}
The Madden-Julian Oscillation (MJO) is a major source of predictability on the sub-seasonal (10- to 90-days) time scale. An improved forecast of the MJO, may have important socioeconomic impacts due to the influence of MJO on both, tropical and extratropical weather extremes. Although in the last decades state-of-the-art climate models have proved their capability for forecasting the MJO exceeding the 5 weeks prediction skill, there is still room for improving the prediction. In

5 this study we use Multiple Linear Regression (MLR) and a Machine Learning (ML) algorithm as post-processing methods to improve the forecast of the model that currently holds the best MJO forecasting performance, the European Centre for Medium-Range Weather Forecast (ECMWF) model. We find that both MLR and ML improve the MJO prediction and that ML outperforms MLR. The largest improvement is in the prediction of the MJO geographical location and intensity.
\end{abstract}

\section{Introduction}

The Madden-Julian Oscillation (MJO) with its 30- to 60-day oscillation is the major sub-seasonal fluctuation in tropical weather (Madden and Julian, 1971, 1972; Vitart, 2009; Lau and Waliser, 2011; Zhang et al., 2013; Ferranti et al., 2018). It is the main source of intra-seasonal fluctuations in the Indian monsoon (Taraphdar et al., 2018; Díaz et al., 2020), and is also known to modulate the tropical cyclogenesis (Camargo et al., 2009; Klotzbach, 2010; Fowler and Pritchard, 2020), to have a two-way interaction with El Niño-Southern Oscillation (ENSO) (Bergman et al., 2001), to influence the Asian-Australian monsoon (Wheeler et al., 2009), and be influenced by the quasi-biennial oscillation (Wang et al., 2019; Martin et al., 2021b). Moreover, the MJO not only affects the tropical weather, but also the extra-tropical weather through teleconnections (Alvarez et al., 2017; Ungerovich et al., 2021). Therefore, MJO has a large impact on the economy, society, and agriculture, motivating the wide interest in its prediction.

Many efforts have been made in this direction in the last decades, with dynamical models leading to the current best forecasts (Jiang et al., 2020), but despite the continuous progress of the dynamical models, there is still room for improvement in 
https://doi.org/10.5194/egusphere-2022-2

Preprint. Discussion started: 7 March 2022

(c) Author(s) 2022. CC BY 4.0 License.

(c) (i)

the MJO prediction (Zhang et al., 2013; Jiang et al., 2020). In particular, an improvement of the prediction skill when MJO crosses the Maritime Continent (MC) barrier (Wu and Hsu, 2009; Kim et al., 2016; Barrett et al., 2021) will be of practical importance due to the influence of MJO on ENSO, as an improved MJO prediction may contribute to improving the prediction of ENSO.

Machine Learning (ML) algorithms are being extensively used in many fields, and they are gaining a foothold in weather and climate forecasts (O'Gorman and Dwyer, 2018; Nooteboom et al., 2018; Dijkstra et al., 2019; Ham et al., 2019; Dasgupta et al., 2020; Tseng et al., 2020; Gagne II et al., 2020; Silini et al., 2021) among many others. Although MJO predictions obtained using ML models do not outperform dynamical models (Silini et al., 2021; Martin et al., 2021a), a hybrid approach, combining dynamical models and ML techniques, may improve the results. In this way, it is possible to use dynamical models that have been developed across decades, based on physical phenomena, in combination with data-driven ML techniques, an approach that has shown its ability to reduce the gap between observations and dynamical models' forecasts (Rasp and Lerch, 2018; McGovern et al., 2019; Scheuerer et al., 2020; Haupt et al., 2021; Vannitsem et al., 2021).

Recently, it has been shown that bias correction in linear dynamics (Wu and Jin, 2021) and the use of Deep Learning (DL) (Kim et al., 2021) can improve the MJO prediction. Specifically, Kim et al. (2021) have shown that the performance of poor models becomes comparable to that of the best model after DL-correction. Here we deal with a related but different problem: can we use a rather simple ML algorithm -a single-hidden layer Feed-forward Neural Network (FFNN)- to improve the forecast of the model that provides the best MJO prediction?

Currently, the best forecast dynamical model in terms of MJO prediction skill is the one developed by the European Centre for Medium-Range Weather Forecast (ECMWF) (Jiang et al., 2020). Therefore, in this study we attempt to improve ECMWF forecasts by using an ANN. We also analyze the performance of Multiple Linear Regression (MLR) as a baseline post-processing tool.

To quantify the forecast skill we use four metrics, namely the bivariate correlation coefficient (COR), the bivariate rootmean-square error (RMSE), with threshold values $\mathrm{COR}=0.5$ and $\mathrm{RMSE}=1.4$, as well as the amplitude error and the phase error (Rashid et al., 2011).

We apply the post-processing ML and MLR techniques to the ensemble mean of ECMWF, and we show that ML outperforms MLR, being able to correct the ECMWF MJO forecasts, and the improvement lasts for longer than four weeks. In particular, ML improves the prediction of the MJO over the MC and its amplitude, while the phase errors obtained with the two postprocessing techniques are similar.

\section{Data, Methods and Models}

\subsection{RMM Data}

For this study, we use the Real-time Multivariate MJO (RMM) index (Wheeler and Hendon, 2004) as labels for the supervised learning method, which is used to characterize the MJO geographical position and intensity. The first two principal components of the combined empirical orthogonal functions (EOFs) of outgoing longwave radiation (OLR), zonal wind at 200 and $850 \mathrm{hPa}$ 
averaged between $15^{\circ} \mathrm{N}$ and $15^{\circ} \mathrm{S}$ are labeled RMM1 and RMM2. With a polar transformation, it is possible to define the MJO phase and amplitude. The phase is divided into 8 classes, each corresponding to a different sector of the phase diagram defining the observed MJO life cycle. The amplitude, describing the MJO intensity, when smaller than 1 defines a non-active MJO. The ERA5 RMM1 and RMM2 from 13th June 1999 to 29th June 2019 were downloaded from ecm (2021). This time window is selected to match the ECMWF reforecasts, presented in the previous section.

\subsection{ECMWF RMM reforecasts}

60 The samples used as input for the ANN and to assess the model performance, are ECMWF reforecasts with Cyrcle 46r1 freely available from ecm (2021). This dataset is composed of 110 initial dates per year for 20 years, between the 13th June 1999 and the 29th June 2019. In total there are 2200 starting dates, from which a 46-lead-days prediction is available. The dataset provides the prediction of four variables: the first two principal components of the RMM index, and their polar transformation. For each starting day and variable there are 12 time series of 46 points. One is the controlled forecast (cf) corresponding to a forecast without any perturbations, then there are 10 perturbed forecasts members (pf) which have slightly different initial conditions from the $\mathrm{cf}$ to take into consideration errors in observations and the chaotic nature of weather. Finally there is the ensemble mean (em), which corresponds to the mean of the 11 members ( $\mathrm{cf}+10 \mathrm{pf}$ ). In this particular study, we made use solely of the em data

\subsection{Prediction skill}

70 To know how good a model is in predicting, we present here the metrics that will be used. For sake of comparison, we use the same metrics adopted in Kim et al. (2018), which are adapted from Lin et al. (2008); Rashid et al. (2011), where they define the COR and RMSE as follows:

$\operatorname{COR}(\tau)=\frac{\sum_{t=1}^{N}\left[a_{1}(t) b_{1}(t, \tau)+a_{2}(t) b_{2}(t, \tau)\right]}{\sqrt{\sum_{t=1}^{N}\left[a_{1}^{2}(t)+a_{2}^{2}(t)\right]} \sqrt{\sum_{t=1}^{N}\left[b_{1}^{2}(t, \tau)+b_{2}^{2}(t, \tau)\right]}}$,

$\operatorname{RMSE}(\tau)=\sqrt{\frac{1}{N} \sum_{t=1}^{N}\left[\left|a_{1}(t)-b_{1}(t, \tau)\right|^{2}+\left|a_{2}(t)-b_{2}(t, \tau)\right|^{2}\right]}$,

where $a_{1}(t)$ and $a_{2}(t)$ correspond to the observed RMM1 and RMM2 at time $t$, while $b_{1}(t, \tau)$ and $b_{2}(t, \tau)$ will be the respective forecasts for time step $t$ for a lead time of $\tau$ days, and $N$ is the number of forecasts. The bivariate correlation coefficient expresses the strength of the linear relationship between the forecasts and the observations, while the root-mean-square error compares the difference between the values of the forecasts and the observations.

In this study we use COR=0.5 and RMSE=1.4 as prediction skill thresholds (Rashid et al., 2011). The RMM prediction skill is defined as the time in which the COR takes a value below 0.5 and RMSE gets above 1.4. For a given lead time, the COR and RMSE are the average value up to that lead time. 
https://doi.org/10.5194/egusphere-2022-2

Preprint. Discussion started: 7 March 2022

(c) Author(s) 2022. CC BY 4.0 License.

(c) (i)

\subsection{Amplitude and phase error}

To characterize the MJO it is convenient to perform a change of coordinates from cartesian to polar (RMM1, RMM2) $\rightarrow(\mathrm{A}, \varphi)$. The MJO amplitude $A(t)$, describing its intensity, can be written as:

$85 A(t)=\sqrt{R M M 1^{2}(t)+R M M 2^{2}(t)}$,

while the MJO phase $\varphi(t)$, describing the geographical position of the enhanced rainfall region center, can be written as:

$\varphi(t)=\tan ^{-1}\left(\frac{R M M 2(t)}{R M M 1(t)}\right)$.

By definition Rashid et al. (2011), the amplitude error for a given lead time $E_{A}(\tau)$ can be expressed as

$E_{A}(\tau)=\frac{1}{N} \sum_{t=1}^{N}\left(A_{\text {pred }}(t, \tau)-A_{o b s}(t)\right)$,

where $N$ represents the number of predicted days, $A_{o b s}(t)$ is the observations amplitude at time $t$ and $A_{\text {pred }}(t, \tau)$ is the predictions amplitude at time $t$ with a lead time of $\tau$ days. The phase error $E_{\varphi}(\tau)$ is defined by

$E_{\varphi}(\tau)=\frac{1}{N} \sum_{t=1}^{N} \tan ^{-1}\left(\frac{a_{1}(t) b_{2}(t, \tau)-a_{2}(t) b_{1}(t, \tau)}{a_{1}(t) b_{1}(t, \tau)}\right)$,

where $a_{1}(t)$ and $a_{2}(t)$ correspond to the observed RMM1 and RMM2 at time $t$, while $b_{1}(t, \tau)$ and $b_{2}(t, \tau)$ correspond to the predicted RMM1 and RMM2 at time $t$ with a lead time of $\tau$ days. These two metrics allow us to analyze in more detail the model performance to predict the MJO, in conjunction with COR and RMSE.

\subsection{Post-processing methods}

The post-processing machine learning tool built for this study is a fully connected feedforward neural network (FFNN) composed of an input layer containing $N_{\text {in }}$ neurons, a single hidden layer with $N_{h}$ neurons, and an output layer with $N_{\text {out }}$ neurons, as shown in Fig 1. The activation function used is the Rectified Linear Unit (ReLU), which transforms the weighted sum of the input values by returning 0 in case of a negative-sum, and the result of the sum otherwise. Dealing with a supervised regression problem, the mean-squared error (MSE) is extensively employed as loss function, and it is used in the framework of this study to compare the neural network output with the observations (labels). An adaptive optimizer (Adam) is selected to automatically manage the learning rate during the training phase.

We use an adaptive number of neurons depending on the number of days we want to forecast. The ECMWF reforecasts provide predictions up to a lead time of 46 days for both RMM1 and RMM2, and we build a different network for each lead time. This means that the number of output neurons $N_{\text {out }}$ can fall between 2 and 92 because we use both RMM1 and RMM2.

After selecting the number of output neurons (which is even and in fact defines our lead time, $\tau=N_{h} / 2$ ), we adapt the number of input $N_{i n}$ and hidden neurons $N_{h}$ as follows. As input, the networks receive the ECMWF reforecasts, which also limit the 


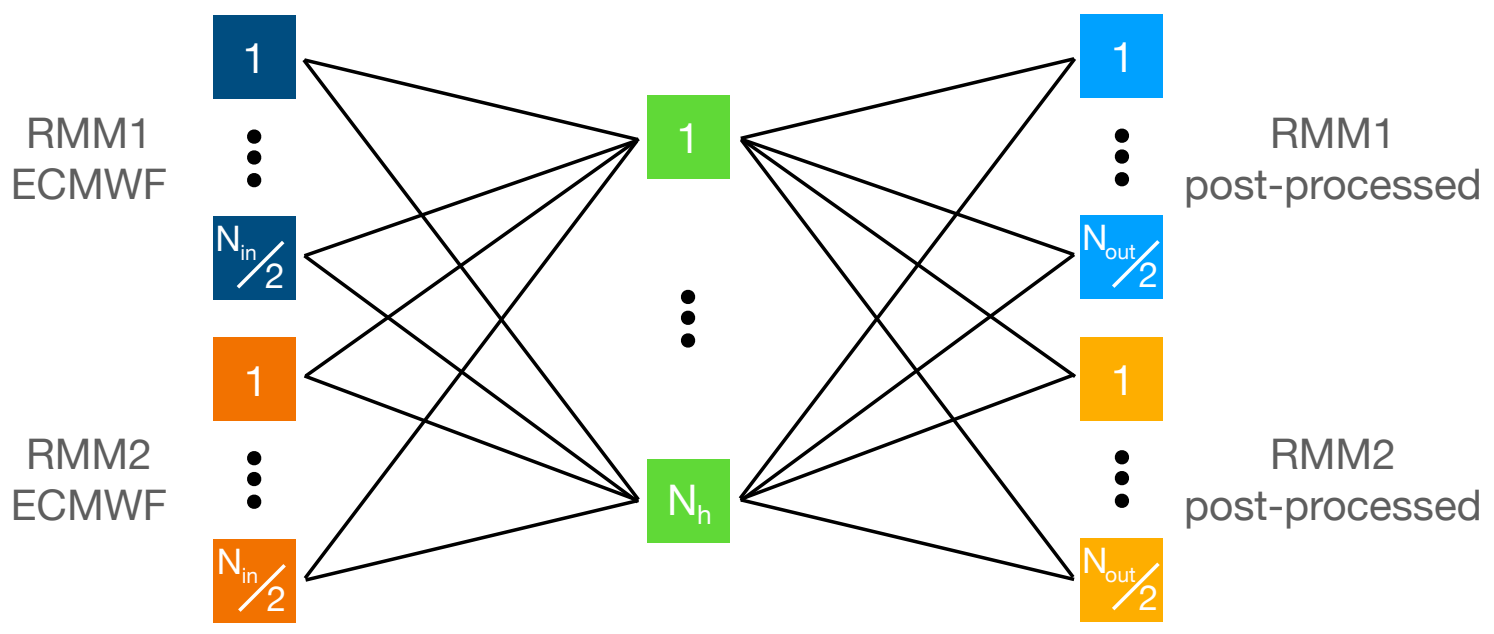

Figure 1. ANN architecture employed for this study.

number of input neurons $N_{i n}$ in the range between 2 and 92 . After training the networks with different $N_{i n}$, we found the best result is obtained with $N_{\text {in }}=N_{\text {out }}+6$ with an upper limit of 92 . Using all 92 inputs, the prediction skill for short lead times slightly decreases. For simplicity, a fixed number of 92 inputs could also be used. An interpretation of the reason behind this result is that to correct the prediction values for a given day, the future predicted values can help up to some extent. To correct the prediction of a given day, for each RMM we use the predicted values of up to 3 days after that particular day. To avoid overfitting, we want the number of hidden neurons to be relatively small, for this reason after some tests, we select $N_{h}=N_{\text {in }} / 2$.

The training has been performed over 100 epochs which allows to not overfit the model, and the model performance is tested using a walk-forward validation, where we found the best minimum number of samples, out of 2200 available, to be 1700 . From the minimum number of samples, the train set is then extended by 100 samples ( $\sim 1$ year) for each run and tested on the subsequent 200 samples ( 2 years). Other methods to avoid overfitting could also be used, such as early stopping or drop-out. The MLR corrects the RMMs separately, using the ECMWF predictions as input and the ERA5 observations as output, with the same walk-forward validation used for the ANNs.

\section{Results}

The first part of this section will be devoted to the results obtained for the MJO amplitude and phase. In the second part we present the prediction skill assessment using the COR 0.5 level, and RMSE 1.4 level as metrics, while in the last part of the section we show how the different forecast methods perform for different MJO initial phases.

The results are obtained training the ANN from 13th of June 1999 using a walk-forward validation, and averaging the error obtained by testing over different unseen time windows from 5th December 2014 to 29th June 2019. The size of the windows is defined by the selected number of initial days from which the ECMWF forecast starts. Due to the bi-weekly acquisition of 

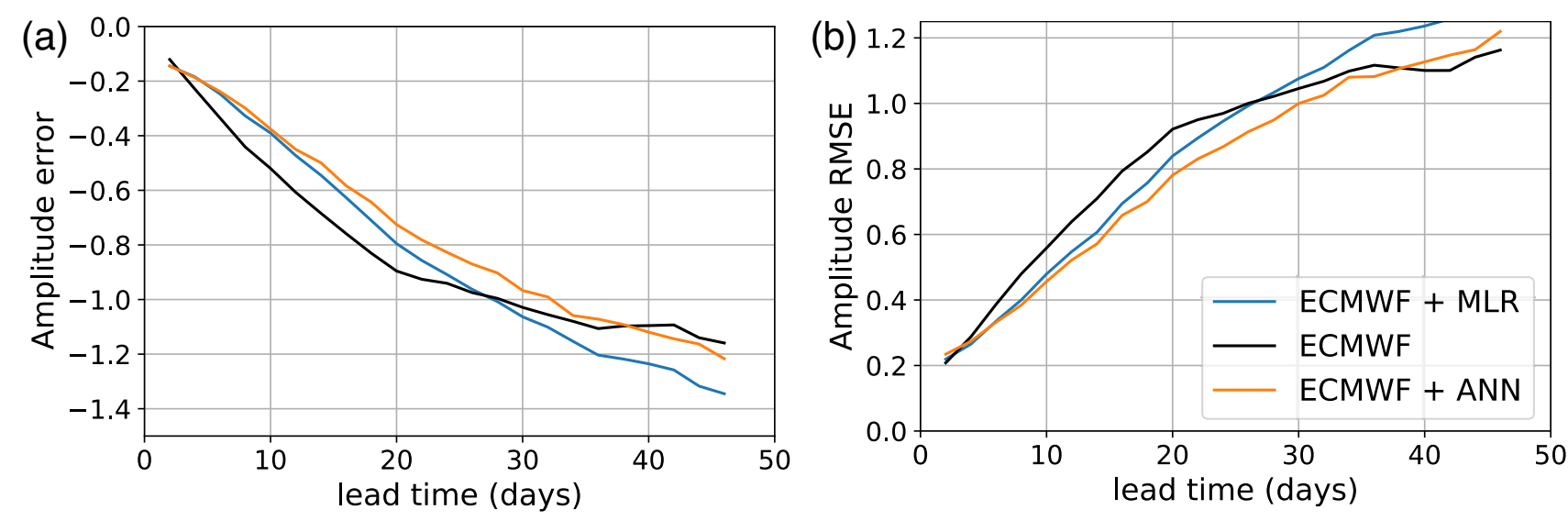

Figure 2. (a) MJO amplitude error and (b) amplitude RMSE (b) as a function of the lead time for events starting with an amplitude larger than 1. The color indicates the forecast model, the black line corresponds to the ECMWF forecast, the blue line corresponds to the MLR correction of the ECMWF forecast, while the orange line corresponds to the post-processed ECMWF forecast with an ANN.

ECMWF, this means that each window of 200 points corresponds to 2 years approximately. Each member of the ensemble over which the average is performed, corresponds to a test set used for the walk-forward validation. Different sizes of the test set between 100 and 500 samples have been tested, leading to prediction skills that vary sensibly. For this reason, it is important to take into account that results may vary depending on the test set and its size, albeit preserving the same general result: the post-processing corrections improve the ECMWF forecasts.

In Fig. 2, we show the error on the MJO amplitude for events starting with an amplitude larger than 1. We can notice an underestimation of the amplitude as expected (Jiang et al., 2020). Nevertheless, the post-processed amplitudes are closer to the observed ones, with respect to the raw ECMWF forecast. The maximum improvement occurs for a lead time of 28 days when the ECMWF-ANN model has a RMSE similar to the RMSE of the uncorrected ECMWF at a lead time of 20 days.

By the definition of the amplitude error, errors of opposite sign could potentially cancel out resulting in misleading conclusions. For this reason in Fig. 2 we also provide the RMSE of the amplitude error, which shows a similar behavior as before. Both post-processing techniques improve the results, with the ANN bringing the highest benefits in terms of the magnitude of error reduction, and the forecasting horizon of the improvement.

In Fig. 3, we present the MJO phase error. The post-processing techniques provide an improved prediction, during which all three models predict a negative phase. A positive phase error indicates a faster propagating MJO, while a negative error represents a slower propagation. The ECMWF forecast shows an overall slower propagation of the MJO with respect to the observations, and both post-processing corrections provides an increment of the MJO speed prediction. In particular, at the 18 days lead time we can notice an increment of the ECMWF phase error, which MLR and ML tend to correct. 


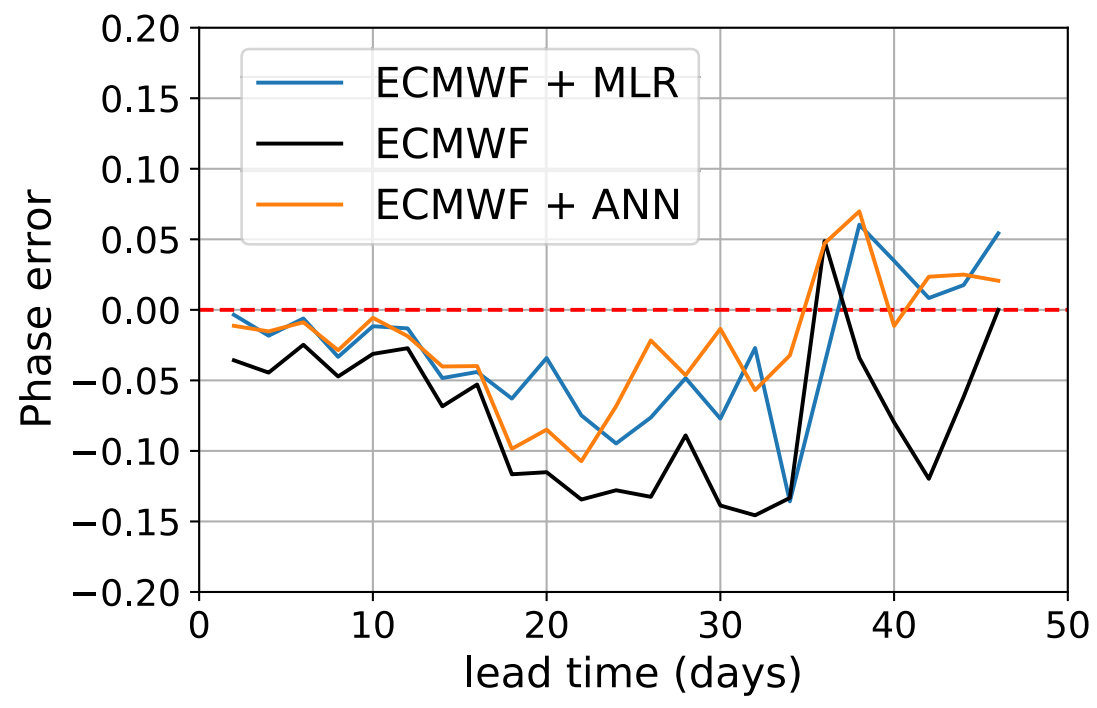

Figure 3. MJO phase error for events starting with an amplitude larger than 1. The color indicates the forecast model, the black line corresponds to the ECMWF forecast, the blue line corresponds to the MLR correction of the ECMWF forecast, while the orange line corresponds to the post-processed ECMWF forecast with an ANN.

Figure 4, shows the COR and RMSE of the ECMWF ensemble mean forecasts, the MLR, and ANN post-processing. A COR of 0.5 is taken here as baseline for useful prediction skill. We see an improvement of the a prediction skill at the $\mathrm{COR}=0.5$ level of about 1 day. However, in terms of RMSE, up to a lead time of 4 weeks, neither post-processing technique crosses the RMSE-threshold of 1.4, and therefore, they both improve the prediction skill with respect to the raw, unprocessed output of the ECMWF model.

In Fig. 5, we display the comparison between the observations, the ECMWF forecast, and its corrections, in a Wheeler-Hendon phase diagram for two different starting dates of the same MJO event. The dots are marked every 7 days to identify the weeks. In the left panel, the 3 weeks prediction starts on the 21st November 2018 and displays its progression from the Western Hemisphere over the Indian Ocean. It is possible to notice that both post-processing techniques display very similar prediction, with a slightly larger amplitude than ECMWF, closer to the observations for all lead times. In the right panel, the 3 weeks prediction starts on the 5th December 2018 in the Indian Ocean. We can see a drop of accuracy in the ECMWF prediction, and the MLR post-processing, approaching the MC. The ML correction instead preserves a larger amplitude, closer to the observations.

It is also possible to notice that while the speed of the MJO event is well predicted in the left panel, in the right one there is a drop of the MJO speed forecast over the Indian Ocean and MC. 

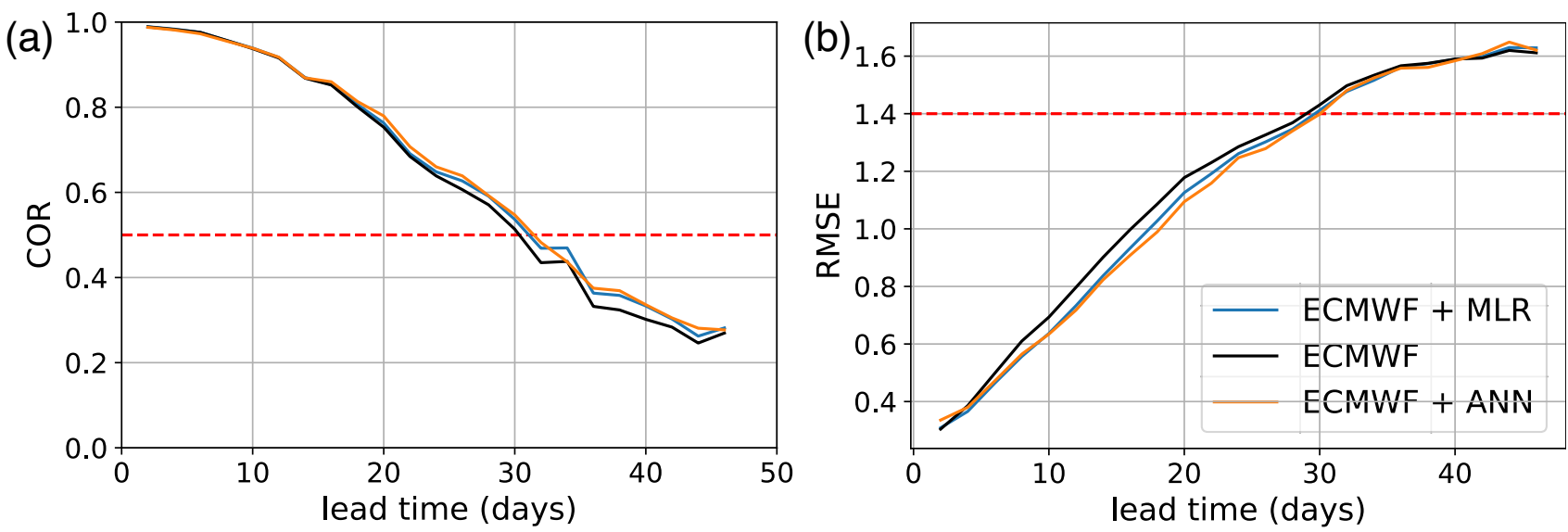

Figure 4. (a) COR and (b) RMSE as a function of the forecast lead time for events starting with an amplitude larger than 1. The color indicates the forecast model and the red dashed line indicates the prediction skill threshold of COR=0.5 and RMSE $=1.4$. The black line corresponds to the ECMWF forecast, the blue line corresponds to the post-processed ECMWF forecast with MLR, while in orange it is shown the post-processed ECMWF forecast with an ANN.

Here we presented an example of a strongly active MJO event, where the corrections clearly improve the ECMWF prediction and it is among the best found. All predictions from the 12th of December 2014 to the 18th of June 2019, can be found in Silini (2021b). Looking at these results it is possible to appreciate the general improvement provided by the post-processing corrections.

Finally we study the amplitude error, the phase error, the COR, and RMSE, as a function of the different initial phases of MJO. As displayed in Fig. 6, applying post-processing methods improves the amplitude error for all initial phases. The MLR provides an improvement with respect to the ECMWF model, but the ML correction leads to the lowest error. Concerning the initial phases, we find the lowest amplitude error when an MJO event starts over the MC, while the largest is found in phase 2, over the Indian Ocean. With the MJO propagating at an average speed of $5 \mathrm{~ms}^{-1}$, events starting in phase 2 will cross the MC in 2-3 weeks time (Kim et al., 2014). The phase error displays a large worsening of the MJO localization prediction, when the forecast starts between the MC and Western Pacific (phase 6-8). This observation is consistent with Fig. 5, where we noticed a drop in the accuracy of the MJO speed prediction over the Indian Ocean and MC. The COR finds its maximum when starting over the MC continent, consistently with the amplitude error. The ML correction has the highest COR except for phase 8, where MLR leads to the highest one. The RMSE is very consistent with the COR, in which we find the the minimum in phase 4, and the ML correction having the lowest error, except for phase 8. Overall, we can conclude that the ML post-processing is worth applying especially to reduce the error on the amplitude prediction, while MLR could be useful for a better prediction of the MJO location. 


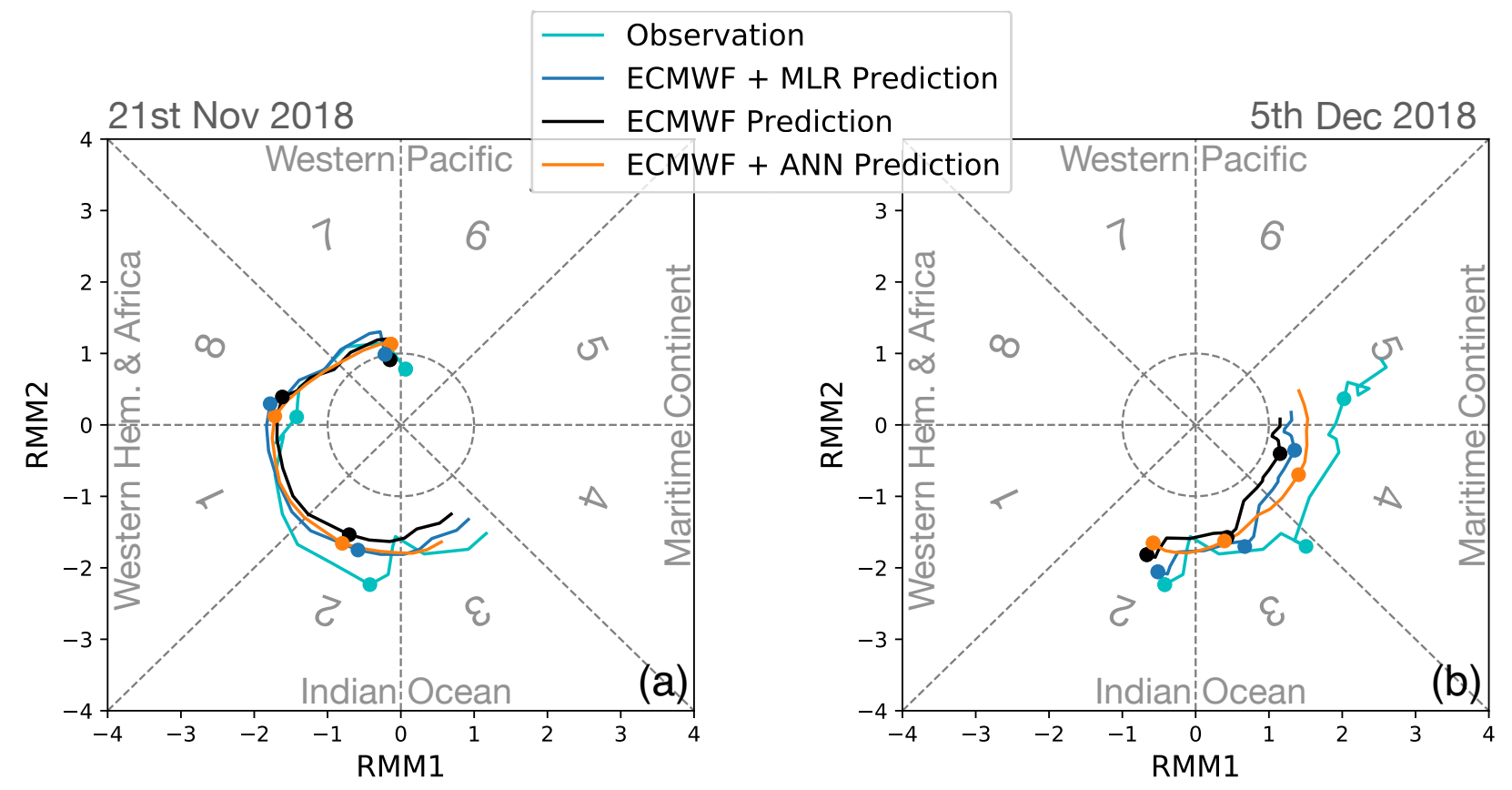

Figure 5. Wheeler-Hendon phase diagram for two different starting dates of the same MJO event, and a 3 weeks prediction. Panel (a) starting date is the 21st November 2018. The MJO enhanced rainfall region travels across the western Hemisphere and Indian Ocean. Panel (b) starting date is the 5th of December 2018, and represents a 3 weeks prediction approaching and traveling over the MC. The rotation of the event in the phase diagram is counter-clockwise, and the dots are included every 7 days, marking the different weeks.

\section{Discussion}

It is interesting to compare the results presented in Fig. 4 with those reported in Fig. 7 of the Supplementary Information in Kim et al. (2021), keeping in mind that Kim et al. show the mean BCOR for the 8 dynamical models considered. While it can be seen in Fig. 7 that for short lead times (up to 2 weeks) a clear improvement with DL-post-processing is obtained, the average BCOR for short lead times is quite low compared to the ECMWF prediction. We can also notice that the improvement obtained by Kim et al. (2021) fades away by the 4th week. In contrast, in our case, for short lead times there is no significant improvement (as it could be expected, due to the fact that ECMWF model provides the best MJO forecast), but our improvement lasts for longer lead times.

It is also interesting to compare the different post-processing approaches used. While we use a feedforward neural network (FFNN) architecture, Kim et al. (2021) used a Deep Learning (DL) network, specifically, a Long Short-Term Memory (LSTM) network. Having a simpler architecture, FFNNs are usually faster to train and to use than LSTMs. While LSTMs have been proven to be powerful for time sequence modeling, as shown in Kim et al. (2021), in our case we are not trying to predict the future of a time series using its past, but we are trying to improve the predictions. 
https://doi.org/10.5194/egusphere-2022-2

Preprint. Discussion started: 7 March 2022

(c) Author(s) 2022. CC BY 4.0 License.

(c) $\underset{\mathrm{BY}}{\mathrm{B}}$

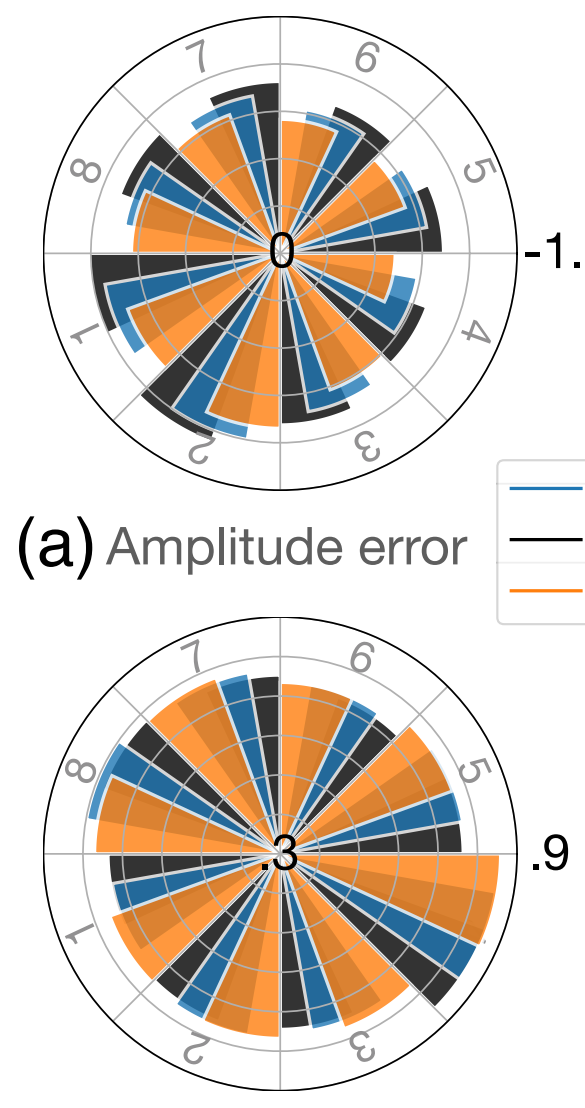

(c) COR
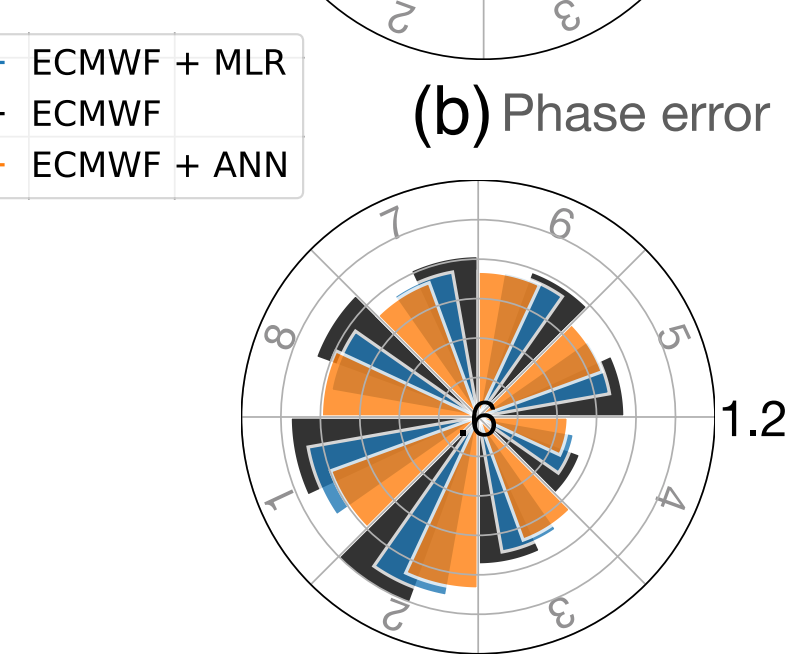

(d) RMSE

Figure 6. (a) Amplitude error, (b) phase error, (c) COR, and (d) RMSE, for the different MJO initial phases, for events starting with an amplitude larger than 1 . The plots show the mean for lead times up to 5 weeks. The different colors represent the different prediction methods. 
There are other differences in the architecture of the networks used: we found that to improve the prediction of the RMMs for a day $t$, the information in the past and future predictions can both help the correction, while in Kim et al. (2021), the future model's predictions (which are available) are not used for the correction. Another difference is that while the algorithm used by Kim et al. (2021) performs an expansion of the system dimensionality (hidden nodes $>$ input nodes), we found good results performing a compression (hidden nodes $<$ input nodes).

Comparing our results to those of post-processing ensemble weather predictions on medium-range time-scales (Vannitsem et al., 2021), we find that the general magnitude of improvements over the predictions of the dynamical model is lower. This indicates the increasingly difficult challenge to obtain accurate MJO predictions for longer lead times, likely due to a generally lower predictability and a lower level of useful information that can be learned from the raw ensemble predictions compared to those of many other weather variables on shorter time scales. That said, our results indicating the potential of modern DL methods to improve over classical statistical approaches are well in line with the findings for medium-range post-processing (Rasp and Lerch, 2018; Vannitsem et al., 2021; Haupt et al., 2021).

\section{Conclusions}

We employed a MLR and a ML algorithm to perform a post-processing correction of the prediction of the dynamical model that currently holds the highest MJO prediction skill (Jiang et al., 2020), developed by ECMWF.

The largest improvement is found in the MJO amplitude and phase individually, which decreases the underestimation of the amplitude, providing a more accurate predicted geographical location of the MJO. The amplitude and phase estimation are improved for all lead times up to 5 weeks.

We obtained an improved prediction skill of about 1 day for a COR of 0.5 .

Plotting the forecasts in a Wheeler-Hendon phase diagram we found an improvement predicting the MJO propagation, notably across the MC, which helps overcome the MC barrier.

Considering the results obtained for each initial MJO phase, we found that both post-processing tools improve the prediction, with the ML correction being the best.

The ML technique provides an improvement over MLR for all initial phases except phase 8 . In the case of phase forecast it might be also sufficient to use MLR instead of ML. This suggests a predominance of linear corrections to improve the MJO phase forecast.

This study confirms the potential of post-processing techniques to reduce the knowledge and bias gap between dynamical models forecasts and observations, providing advancement in MJO prediction.

As future work, it would be interesting to test a stochastic approach to post-processing (as in Rasp and Lerch (2018), which would allow to obtain a probabilistic forecast

Although the improvement provided by the MLR and ML techniques, a post-processing method will always strongly rely on the accuracy of the dynamical model's forecasts. For this reason, it is crucial to work on both dynamical models and machine learning methods to progress. 
https://doi.org/10.5194/egusphere-2022-2

Preprint. Discussion started: 7 March 2022

(c) Author(s) 2022. CC BY 4.0 License.

(c) (i)

Code and data availability. The Keras TensorFlow Abadi et al. (2015) trained FFNN can be found in Silini (2021a). The RMM data, and the ECMWF reforecasts can be freely downloaded from ecm (2021).

Author contributions. R. S. performed the analysis and prepared the figures; R. S. and S. L. and N. M. designed the study; all authors discussed the results, wrote and reviewed the manuscript

Competing interests. The authors declare no competing interests.

Acknowledgements. The authors thank Linus Magnusson for providing access to the data. This work received funding from the European Union's Horizon 2020 research and innovation programme under the Marie Skłodowska Curie Grant Agreement No 813844. C.M. also acknowledges funding by the Spanish Ministerio de Ciencia, Innovacion y Universidades (PGC2018-099443-B-I00) and the ICREA ACADEMIA program of Generalitat de Catalunya. S.L. acknowledges support by the Vector Stiftung through the Young Investigator Group "Artificial Intelligence for Probabilistic Weather Forecasting". 
https://doi.org/10.5194/egusphere-2022-2

Preprint. Discussion started: 7 March 2022

(c) Author(s) 2022. CC BY 4.0 License.

(c) (i)

\section{References}

ECMWF RMM reforecasts data, https://acquisition.ecmwf.int/ecpds/data/list/RMMS/ecmwf/reforecasts/, accessed: $2021-02,2021$.

Abadi, M., Agarwal, A., Barham, P., Brevdo, E., Chen, Z., Citro, C., Corrado, G. S., Davis, A., Dean, J., Devin, M., Ghemawat, S., Goodfellow, I., Harp, A., Irving, G., Isard, M., Jia, Y., Jozefowicz, R., Kaiser, L., Kudlur, M., Levenberg, J., Mané, D., Monga, R., Moore, S., Murray, D., Olah, C., Schuster, M., Shlens, J., Steiner, B., Sutskever, I., Talwar, K., Tucker, P., Vanhoucke, V., Vasudevan, V., Viégas, F., Vinyals, O., Warden, P., Wattenberg, M., Wicke, M., Yu, Y., and Zheng, X.: TensorFlow: Large-Scale Machine Learning on Heterogeneous Systems, http://tensorflow.org/, software available from tensorflow.org, 2015.

Alvarez, M. S., Vera, C. S., and Kiladis, G. N.: MJO Modulating the Activity of the Leading Mode of Intraseasonal Variability in South America, Atmosphere, 8, https://doi.org/https://doi.org/10.3390/atmos8120232, 2017.

Barrett, B. S., Densmore, C. R., Ray, P., and Sanabia, E. R.: Active and weakening MJO events in the Maritime Continent, Climate Dynamics, https://doi.org/https://doi.org/10.1007/s00382-021-05699-8, 2021.

Bergman, J. W., Hendon, H. H., and Weickmann, K. M.: Intraseasonal Air-Sea Interactions at the Onset of El Niño, Journal of Climate, 14, 1702-1719, 2001.

Camargo, S. J., Wheeler, M. C., and Sobel, A. H.: Diagnosis of the MJO Modulation of Tropical Cyclogenesis Using an Empirical Index, Journal of the Atmospheric Sciences, 66, 3061 - 3074, https://doi.org/https://doi.org/10.1175/2009JAS3101.1, 2009.

Dasgupta, P., Metya, A., Naidu, C. V., Singh, M., and Roxy, M. K.: Exploring the long-term changes in the Madden Julian Oscillation using machine learning, Scientific Reports, 10, 18 567, https://doi.org/https://doi.org/10.1038/s41598-020-75508-5, 2020.

Díaz, N., Barreiro, M., and Rubido, N.: Intraseasonal Predictions for the South American Rainfall Dipole, Geophysical Research Letters, 47 , https://doi.org/https://doi.org/10.1029/2020GL089985, 2020.

Dijkstra, H. A., Petersik, P., Hernández-García, E., and López, C.: The Application of Machine Learning Techniques to Improve El Niño Prediction Skill, Frontiers in Physics, 7, 153, https://doi.org/https://doi.org/10.3389/fphy.2019.00153, 2019.

Ferranti, L., Magnusson, L., Vitart, F., and Richardson, D. S.: How far in advance can we predict changes in large-scale flow leading to severe cold conditions over Europe?, Quarterly Journal of the Royal Meteorological Society, 144, 1788-1802, https://doi.org/https://doi.org/10.1002/qj.3341, 2018.

Fowler, M. D. and Pritchard, M. S.: Regional MJO Modulation of Northwest Pacific Tropical Cyclones Driven by Multiple Transient Controls, Geophysical Research Letters, 47, e2020GL087 148, https://doi.org/https://doi.org/10.1029/2020GL087148, 2020.

Gagne II, D. J., Christensen, H. M., Subramanian, A. C., and Monahan, A. H.: Machine Learning for Stochastic Parameterization: Generative Adversarial Networks in the Lorenz '96 Model, Journal of Advances in Modeling Earth Systems, 12, e2019MS001 896, https://doi.org/https://doi.org/10.1029/2019MS001896, 2020.

Ham, Y.-G., Kim, J.-H., and Luo, J.-J.: Deep learning for multi-year ENSO forecasts, Nature, 573, 568-572, https://doi.org/https://doi.org/10.1038/s41586-019-1559-7, 2019.

265 Haupt, S. E., Chapman, W., Adams, S. V., Kirkwood, C., Hosking, J. S., Robinson, N. H., Lerch, S., and Subramanian, A. C.: Towards implementing artificial intelligence post-processing in weather and climate: proposed actions from the Oxford 2019 workshop, Philosophical Transactions of the Royal Society A: Mathematical, Physical and Engineering Sciences, 379, 20200091, https://doi.org/https://doi.org/10.1098/rsta.2020.0091, 2021. 
https://doi.org/10.5194/egusphere-2022-2

Preprint. Discussion started: 7 March 2022

(c) Author(s) 2022. CC BY 4.0 License.

\section{(c) (i)}

Jiang, X., Adames, A. F., Kim, D., Maloney, E. D., Lin, H., Kim, H., Zhang, C., DeMott, C. A., and Klingaman, N. P.: Fifty Years of Research on the Madden-Julian Oscillation: Recent Progress, Challenges, and Perspectives, Journal of Geophysical Research: Atmospheres, 125, e2019JD030 911, https://doi.org/https://doi.org/10.1029/2019JD030911, 2020.

Kim, H., Vitart, F., and Waliser, D. E.: Prediction of the Madden-Julian Oscillation: A Review, Journal of Climate, 31 , 9425 - 9443 , https://doi.org/https://doi.org/10.1175/JCLI-D-18-0210.1, 2018.

Kim, H., Ham, Y. G., Joo, Y. S., and Son, S. W.: Deep learning for bias correction of MJO prediction, Nature Communications, 12, https://doi.org/https://doi.org/10.1038/s41467-021-23406-3, 2021.

Kim, H.-M., Webster, P. J., Toma, V. E., and Kim, D.: Predictability and Prediction Skill of the MJO in Two Operational Forecasting Systems, Journal of Climate, 27, 5364 - 5378, https://doi.org/10.1175/JCLI-D-13-00480.1, 2014.

Kim, H.-M., Kim, D., Vitart, F., Toma, V. E., Kug, J.-S., and Webster, P. J.: MJO Propagation across the Maritime Continent in the ECMWF Ensemble Prediction System, Journal of Climate, 29, 3973-3988, https://doi.org/https://doi.org/10.1175/JCLI-D-15-0862.1, 2016.

Klotzbach, P. J.: On the Madden-Julian Oscillation-Atlantic Hurricane Relationship, Journal of Climate, 23, 282-293, https://doi.org/https://doi.org/10.1175/2009JCLI2978.1, 2010.

Lau, W. K. M. and Waliser, D. E.: Predictability and forecasting, Springer Berlin Heidelberg, Berlin, Heidelberg, https://doi.org/https://doi.org/10.1007/978-3-642-13914-7_12, 2011.

Lin, H., Brunet, G., and Derome, J.: Forecast Skill of the Madden-Julian Oscillation in Two Canadian Atmospheric Models, Monthly Weather Review, 136, 4130 - 4149, https://doi.org/https://doi.org/10.1175/2008MWR2459.1, 2008.

Madden, R. A. and Julian, P. R.: Detection of a 40-50 Day Oscillation in the Zonal Wind in the Tropical Pacific, Journal of Atmospheric Sciences, 28, 702 - 708, https://doi.org/https://doi.org/10.1175/1520-0469(1971)028<0702:DOADOI>2.0.CO;2, 1971.

Madden, R. A. and Julian, P. R.: Description of Global-Scale Circulation Cells in the Tropics with a 40-50 Day Period, Journal of Atmospheric Sciences, 29, 1109 - 1123, https://doi.org/https://doi.org/10.1175/1520-0469(1972)029<1109:DOGSCC>2.0.CO;2, 1972.

Martin, Z. K., Barnes, E. A., and Maloney, E. D.: Using simple, explainable neural networks to predict the Madden-Julian oscillation, Earth and Space Science Open Archive, https://doi.org/https://doi.org/10.1002/essoar.10507439.1, 2021a.

Martin, Z. K., Son, S.-W., Butler, A., Hendon, H., Kim, H., Sobel, A., Yoden, S., and Zhang, C.: The influence of the quasi-biennial oscillation on the Madden-Julian oscillation, Nature Reviews Earth and Environment, pp. 477-489, https://doi.org/https://doi.org/10.1038/s43017021-00173-9, 2021b.

McGovern, A., Lagerquist, R., II, D. J. G., Jergensen, G. E., Elmore, K. L., Homeyer, C. R., and Smith, T.: Making the Black Box More Transparent: Understanding the Physical Implications of Machine Learning, Bulletin of the American Meteorological Society, 100, 21752199, https://doi.org/https://doi.org/10.1175/BAMS-D-18-0195.1, 2019.

Nooteboom, P. D., Feng, Q. Y., López, C., Hernández-García, E., and Dijkstra, H. A.: Using network theory and machine learning to predict El Niño, Earth System Dynamics, 9, 969-983, https://doi.org/https://doi.org/10.5194/esd-9-969-2018, 2018.

O'Gorman, P. A. and Dwyer, J. G.: Using Machine Learning to Parameterize Moist Convection: Potential for Modeling of Climate, Climate Change, and Extreme Events, Journal of Advances in Modeling Earth Systems, 10, 2548-2563, https://doi.org/https://doi.org/10.1029/2018MS001351, 2018.

Rashid, H. A., Hendon, H. H., Wheeler, M. C., and Alves, O.: Prediction of the Madden-Julian oscillation with the POAMA dynamical prediction system, Climate Dynamics, 36, 649-661, https://doi.org/https://doi.org/10.1007/s00382-010-0754-x, 2011.

305 Rasp, S. and Lerch, S.: Neural networks for postprocessing ensemble weather forecasts, Monthly Weather Review, 146, 3885-3900, https://doi.org/https://doi.org/10.1175/MWR-D-18-0187.1, 2018. 
https://doi.org/10.5194/egusphere-2022-2

Preprint. Discussion started: 7 March 2022

(c) Author(s) 2022. CC BY 4.0 License.

(c) (i)

Scheuerer, M., Switanek, M. B., Worsnop, R. P., and Hamill, T. M.: Using Artificial Neural Networks for Generating Probabilistic Subseasonal Precipitation Forecasts over California, Monthly Weather Review, 148, 3489-3506, https://doi.org/https://doi.org/10.1175/MWRD-20-0096.1, 2020.

310 Silini, R.: Feed-forward neural networks, https://doi.org/https://doi.org/10.5281/zenodo.5801453, 2021a.

Silini, R.: Wheeler-Hendon phase diagrams, https://doi.org/https://doi.org/10.5281/zenodo.5801415, $2021 \mathrm{~b}$.

Silini, R., Barreiro, M., and Masoller, C.: Machine learning prediction of the Madden-Julian Oscillation, npj Climate and Atmospheric Science, 4, https://doi.org/https://doi.org/10.1038/s41612-021-00214-6, 2021.

Taraphdar, S., Zhang, F., Leung, L. R., Chen, X., and Pauluis, O. M.: MJO affects the Monsoon Onset Timing Over the Indian Region, Geophysical Research Letters, 45, https://doi.org/https://doi.org/10.1029/2018GL078804, 2018.

Tseng, K.-C., Barnes, E. A., and Maloney, E.: The Importance of Past MJO Activity in Determining the Future State of the Midlatitude Circulation, Journal of Climate, 33, 2131 - 2147, https://doi.org/https://doi.org/10.1175/JCLI-D-19-0512.1, 2020.

Ungerovich, M., Barreiro, M., and Masoller, C.: Influence of Madden-Julian Oscillation on extreme rainfall events in Spring in southern Uruguay, International Journal of Climatology, pp. 1-13, https://doi.org/https://doi.org/10.1002/joc.7022, 2021.

Vannitsem, S., Bremnes, J. B., Demaeyer, J., Evans, G. R., Flowerdew, J., Hemri, S., Lerch, S., Roberts, N., Theis, S., Atencia, A., Bouallègue, Z. B., Bhend, J., Dabernig, M., Cruz, L. D., Hieta, L., Mestre, O., Moret, L., Plenković, I., Schmeits, M., Taillardat, M., den Bergh, J. V., Schaeybroeck, B. V., Whan, K., and Ylhaisi, J.: Statistical Postprocessing for Weather Forecasts: Review, Challenges, and Avenues in a Big Data World, Bulletin of the American Meteorological Society, 102, E681-E699, https://doi.org/https://doi.org/10.1175/BAMS-D-190308.1, 2021.

Vitart, F.: Impact of the Madden Julian Oscillation on tropical storms and risk of landfall in the ECMWF forecast system, Geophysical Research Letters, 36, https://doi.org/https://doi.org/10.1029/2009GL039089, 2009.

Wang, S., Tippett, M. K., Sobel, A. H., Martin, Z. K., and Vitart, F.: Impact of the QBO on Prediction and Predictability of the MJO Convection, Journal of Geophysical Research: Atmospheres, 124, 11 766-11 782, https://doi.org/https://doi.org/10.1029/2019JD030575, 2019.

Wheeler, M. C. and Hendon, H. H.: An All-Season Real-Time Multivariate MJO Index: Development of an Index for Monitoring and Prediction, Monthly Weather Review, 132, 1917 - 1932, https://doi.org/https://doi.org/10.1175/15200493(2004)132<1917:AARMMI>2.0.CO;2, 2004.

Wheeler, M. C., Hendon, H. H., Cleland, S., Meinke, H., and Donald, A.: Impacts of the Madden-Julian Oscillation on Australian Rainfall and Circulation, Journal of Climate, 22, 1482-1498, https://doi.org/https://doi.org/10.1175/2008JCLI2595.1, 2009.

Wu, C.-H. and Hsu, H.-H.: Topographic Influence on the MJO in hte Maritime Continent, Journal of Climate, 22, 5433-5448, https://doi.org/https://doi.org/10.1175/2009JCLI2825.1, 2009.

Wu, J. and Jin, F.-F.: Improving the MJO Forecast of S2S Operation Models by Correcting Their Biases in Linear Dynamics, Geophysical Research Letters, 48, e2020GL091 930, https://doi.org/https://doi.org/10.1029/2020GL091930, 2021.

Zhang, C., Gottschalck, J., Maloney, E. D., Moncrieff, M. W., Vitart, F., Waliser, D. E., Wang, B., and Wheeler, M. C.: Cracking the MJO nut, Geophysical Research Letters, 40, 1223-1230, https://doi.org/https://doi.org/10.1002/grl.50244, 2013. 